\title{
BMJ Open Cross-sectional study evaluating the seroprevalence of SARS-CoV-2 antibodies among healthcare workers and factors associated with exposure during the first wave of the COVID-19 pandemic in New York
}

Alexander Bryan (1D , ${ }^{1,2}$ Kathleen Tatem, ${ }^{2}$ Jillian Diuguid-Gerber, ${ }^{1,3}$ Caroline Cooke, ${ }^{2}$ Anya Romanoff, ${ }^{4,5}$ Nandini Choudhury, ${ }^{6}$ Michael Scanlon, ${ }^{7}$ Preeti Kishore, ${ }^{8}$ Elana Sydney, ${ }^{8}$ Joseph Masci, ${ }^{9,10}$ Parampreet Bakshi, ${ }^{10,11}$ Sahithi Pemmasani, ${ }^{12}$ Nichola J Davis, ${ }^{2}$ Duncan Maru (10) ${ }^{13,14}$

To cite: Bryan A, Tatem K, Diuguid-Gerber J, et al. Crosssectional study evaluating the seroprevalence of SARS-CoV-2 antibodies among healthcare workers and factors associated with exposure during the first wave of the COVID-19 pandemic in New York. BMJ Open 2021;11:e053158. doi:10.1136/ bmjopen-2021-053158

- Prepublication history and additional supplemental material for this paper are available online. To view these files, please visit the journal online (http://dx.doi.org/10.1136/ bmjopen-2021-053158).

Received 07 May 2021 Accepted 18 0ctober 2021

D) Check for updates

(c) Author(s) (or their employer(s)) 2021. Re-use permitted under CC BY-NC. No commercial re-use. See rights and permissions. Published by BMJ.

For numbered affiliations see end of article.

Correspondence to Dr Alexander Bryan; Alexander.Bryan@nyulangone. org

\section{ABSTRACT}

Objective Estimate the seroprevalence of SARS-CoV-2 antibodies among New York City Health and Hospitals $(\mathrm{NYC} \mathrm{H}+\mathrm{H})$ healthcare workers during the first wave of the COVID-19 pandemic, and describe demographic and occupational factors associated with SARS-CoV-2 antibodies among healthcare workers.

Design Descriptive, observational, cross-sectional study using a convenience sample of data from SARS-CoV-2 serological tests accompanied by a demographic and occupational survey administered to healthcare workers. Setting A large, urban public healthcare system in NYC. Participants Participants were employed by NYC $\mathrm{H}+\mathrm{H}$ and either completed serological testing at $\mathrm{NYC} \mathrm{H}+\mathrm{H}$ between 30 April 2020 and 30 June 2020, or completed SARSCoV-2 antibody testing outside of NYC $\mathrm{H}+\mathrm{H}$ and were able to self-report results from the same time period.

Primary outcome measure SARS-CoV-2 serostatus, stratified by key demographic and occupational characteristics reported through the demographic and occupational survey.

Results Seven hundred and twenty-seven survey respondents were included in analysis. Participants had a mean age of 46 years $(S D=12.19)$ and $543(75 \%)$ were women. Two hundred and fourteen (29\%) participants tested positive or reported testing positive for the presence of SARS-CoV-2 antibodies (lgG+). Characteristics associated with positive SARS-CoV-2 serostatus were Black race $(25 \% \lg G+v s 15 \% \lg G-, p=0.001)$, having someone in the household with COVID-19 symptoms (49\% IgG +vs $21 \% \lg G-, p<0.001)$, or having a confirmed COVID-19 case in the household ( $25 \% \lg G+v s 5 \% \lg G-$, $p<0.001)$. Characteristics associated with negative SARSCoV-2 serostatus included working on a COVID-19 patient floor $(27 \% \lg G+v s 36 \% \lg G-, p=0.02)$, working in the intensive care unit $(20 \% \lg G+v s 28 \% \lg G-, p=0.03)$, being employed in a clinical occupation $(64 \% \lg G+v s 78 \%$ IgG-, $\mathrm{p}<0.001)$ or having close contact with a patient with COVID-19 $(51 \% \lg G+v s 62 \% \lg G-, p=0.03)$.
Strengths and limitations of this study

- Strengths of this study included the sampling of a wide range of employees and healthcare occupations, and the utilisation of a detailed survey that allowed for stratification of SARS-CoV-2 serostatus by a wide range of demographic, occupational and exposure factors.

- Limitations of this study include the use of convenience sampling for enrollment, which we attempted to partially mitigate by comparing aggregate participant demographics with overall employee demographics.

- We were also unable to determine when employees with positive SARS-CoV-2 antibodies were infected, which limited interpretation of some of the data.

Conclusions Results underscore the significance that community factors and inequities might have on SARSCoV-2 exposure for healthcare workers.

\section{BACKGROUND}

By the end of 2020, there were almost 85 million confirmed cases of SARS-CoV-2 and over 1.8 million deaths globally. ${ }^{1}$ The pandemic has placed enormous strains on healthcare systems and healthcare workers (HCWs), including inpatient and community-based care providers as well as hospital administrators and support staff. As shown during prior infectious disease outbreaks, protecting HCW through adequate infection control and access to personal protective equipment (PPE) alongside general public health and preventative measures is critical to global pandemic response. ${ }^{2}$ In many countries, however, the current COVID-19 pandemic has 
led to truly unprecedented conditions for HCW and their physical and mental well-being. ${ }^{3}$

Epidemiological and serological data on SARS-CoV-2 among HCW are essential to guide healthcare systems and public health policies and protect HCW. ${ }^{4}$ Early data from China suggested that HCW were at high risk of SARS-CoV-2 infection, ${ }^{5}$ and since then a significant body of literature has emerged on SARS-CoV-2 among HCW. ${ }^{67}$ A systematic review and meta-analysis of 97 studies up through 8 July 2020 including 230398 HCW found a pooled SARS-CoV-2 prevalence rate of $11 \%$ in studies using reverse transcription-PCR tests and 7\% using serum antibody tests, but there were insufficient data in most studies to assess risk factors and exposure levels. ${ }^{7} \mathrm{HCWs}$ are at risk of occupational transmission of SARS-CoV-2 in inpatient and outpatient settings, particularly with inadequate PPE or infection control procedures. ${ }^{89} \mathrm{HCWs}$ are also at risk for community transmission of SARS-CoV-2 ${ }^{10}$ while household members of HCW may be at higher risk compared with the general public. ${ }^{11}$

Among HCW, the risk of infection varies by demographic characteristics, cadre of HCW and work location, with systemic racism playing a clear role in inequities. ${ }^{12}$ Additionally, among HCW with a job setting reported, most infections were associated with nursing and residential care facilities $(67 \%)$ compared with hospital settings (18\%). There are few data on SARS-CoV-2 among communitybased HCW and other social service workers who may have different demographic and occupational risk profiles. ${ }^{13}$

Approximately 6 weeks into the pandemic, the largest public hospital system in the USA, New York City Health and Hospitals (NYC $\mathrm{H}+\mathrm{H})$, initiated universal, voluntary serological testing among all employees. We invited employees who were undergoing serological testing to participate in a survey to assess demographic and occupational factors associated with serostatus. Specifically, we aimed to: (1) estimate the seroprevalence of SARSCoV-2 antibodies among NYC H+H HCW and (2) identify demographic and occupational factors associated with SARS-CoV-2 antibodies among NYC $\mathrm{H}+\mathrm{H}$ HCW during the first wave of the COVID-19 pandemic.

\section{METHODS}

\section{Study setting}

NYC $\mathrm{H}+\mathrm{H}$ employs over 40000 people in a wide range of clinical and non-clinical positions at 11 acute care hospitals and more than 70 community facilities across the city's five boroughs. This study leveraged universal, voluntary SARS-CoV-2 antibody testing which was available at NYC $\mathrm{H}+\mathrm{H}$ during the first wave of the COVID-19 pandemic starting in April 2020. Testing was open to all employees of NYC $\mathrm{H}+\mathrm{H}$ and was available at $\mathrm{NYC} \mathrm{H}+\mathrm{H}$ ambulatory settings across the city.

\section{STUDY DESIGN}

This was an observational, cross-sectional study using data from SARS-CoV-2 serological tests accompanied by a demographic and occupational survey administered to $\mathrm{HCW}$ at NYC $\mathrm{H}+\mathrm{H}$. The primary endpoint was SARS-CoV-2 serological testing outcome, stratified by key demographic and occupational characteristics reported through the survey.

Patients or the public were not involved in the design, or conduct, or reporting, or dissemination plans of our research.

To assess the risk of contracting COVID-19, we developed a modified survey based on the WHO's Protocol for assessment of potential risk factors for 2019-novel coronavirus (2019-nCoV) infection among HCWs in a healthcare setting. ${ }^{4}$ Surveys were self-administered in paper form at testing sites or electronically through a REDCap (Research Electronic Data Capture) survey online. Survey respondents who completed SARS-CoV-2 antibody testing at NYC $\mathrm{H}+\mathrm{H}$ were matched to serological results in the NYC $\mathrm{H}+\mathrm{H}$ electronic health record (EHR). Results of SARS-CoV-2 rtPCR testing were included in secondary analyses when available.

\section{STUDY POPULATION}

Surveys were self-administered in paper form at testing sites, and an electronic version of the survey was emailed system-wide to all NYC $\mathrm{H}+\mathrm{H}$ employees. All NYC $\mathrm{H}+\mathrm{H}$ employees who had serological testing from 30 April 2020 to 30 June 2020 were invited to participate. Additionally, employees who received antibody testing outside of NYC $\mathrm{H}+\mathrm{H}$ between 30 April 2020and 30 June 2020 could complete the survey and self-report serology results.

In order to be eligible for the study, participants needed to meet the following criteria:

1. Employed by NYC $\mathrm{H}+\mathrm{H}$ and either (A) completed serological testing at NYC $\mathrm{H}+\mathrm{H}$ or (B) completed SARSCoV-2 antibody testing outside of NYC $\mathrm{H}+\mathrm{H}$ and were able to self-report results.

2. 18 years of age or older.

3. Capable of providing consent to participate in the study, including English or Spanish fluency.

Additionally, limited data on demographics and seropositivity are included on all $\mathrm{H}+\mathrm{H}$ employees who completed antibody testing from 30 April 2020 to 30 June 2020 but did not participate in the survey study.

\section{KEY DEFINITIONS}

\section{SARS-CoV-2 antibody status}

SARS-Cov-2 antibody status was assigned based on serological results from the NYC H+H EHR. Serological testing at NYC $\mathrm{H}+\mathrm{H}$ was performed on Abbott i1000SR laboratory instruments using the ARCHITECT SARS-CoV-2 IgG chemiluminescence assay from Abbott Laboratories.

For participants who completed serological testing at an outside institution, self-reported results were used. Selfreported results were not independently verified. Under the Emergency Use Authorisation (EUA) issued on 4 February 2020 by the Secretary of the US Department 
of Health and Human Services, immunoassay tests could be offered based on manufacturer-reported data without formal U.S. Food and Drug Administration (FDA) clearance. ${ }^{14}$ On 4 May 2020, the FDA issued an updated policy recommending a minimum sensitivity of $90 \%$ and specificity of $95 \%$ for EUA. ${ }^{15}$

\section{Demographic characteristics}

Demographic characteristics were self-reported. Sex was captured as male or female. Age was calculated from date of birth, and country of origin analysed as US born or non-US born. Participant zip code of primary residence was used to determine borough (ie, Manhattan, Brooklyn, Bronx, Queens, Staten Island, non-NYC). Race and ethnicity were reported separately and variables were combined together into one variable with mutually exclusive categories for analysis, with black representing non-Hispanic black participants and white representing non-Hispanic white participants.

\section{Occupation}

Participants selected occupation from a prespecified list or selected 'other' and provided a free text response answer. Free-text response answers were regrouped into existing or new categories when appropriate. The following additional groupings emerged: care coordination, pharmacy and counselling (distinct from social worker).

\section{STATISTICAL ANALYSIS}

We used descriptive statistics to explore demographic characteristics, case exposure, occupational setting and SARS-CoV-2 serological test results of the study population, and explored the association of serological test results with demographic (eg, zip code) and occupational characteristics and exposures. Continuous measures were expressed as means and SD, categorical variables as counts and proportions. We conducted bivariate analyses using Fisher's exact test for categorical variables and t-tests, as appropriate, with significance set at $\alpha=0.05$. In addition, subsequent $\chi^{2}$ tests were conducted for age using 21-34 as a reference group and for race using white as a reference group. Missing values were removed for all calculations resulting in differing denominators for each demographic question and exposure-related variable. $\mathrm{R}$ Studio V.1.3.1093 using the tidyverse and gmodels packages was used for statistical analysis.

\section{RESULTS}

In total, 19107 staff completed antibody testing at NYC $\mathrm{H}+\mathrm{H}$ from 30 April to 30 June. During that time, there were 1671 respondents to the demographic and occupational survey, and 727 survey respondents were matched to SARS-CoV-2 antibody results, either through the NYC $\mathrm{H}+\mathrm{H}$ EHR or through self-report. Reasons for being unable to match survey respondents to serological results included surveys missing full name or date of birth $(\mathrm{n}=766)$, or name and date of birth match not found in EHR $(n=178)$.

Participants had a mean age of 45.97 years $(\mathrm{SD}=12.19)$ and $543(75 \%)$ were women. Two hundred and sixty-four $(36 \%)$ self-reported as white, $146(20 \%)$ as Hispanic, 128 $(18 \%)$ as black, $148(20 \%)$ as Asian, $19(2.6 \%)$ as other, $11(1.5 \%)$ as multiracial, $4(0.6 \%)$ as Pacific Islander and $6(0.8 \%)$ as missing. Of the 705 participants who reported their country of origin, $322(46 \%)$ were US-born. Over 70 different countries of origin were reported. A total of $539(75 \%)$ participants reported living in NYC. Over half of the sample, $407(58 \%)$ participants, reported having known close contact with at least 1 SARS-CoV-2 positive patient, $254(37 \%)$ noted close contact with materials of at least 1 SARS-CoV-2 positive patient, 210 (29\%) reported living with someone experiencing COVID-19 symptoms and $76(11 \%)$ reported living with someone with confirmed COVID-19.

Compared with all $\mathrm{H}+\mathrm{H}$ staff who completed serology testing between 30 April 20 and 30 June 2020 (n=19107), and the overall $\mathrm{H}+\mathrm{H}$ workforce (estimated 42 000), study participants were similar in sex (female $75 \%$ survey sample vs $70 \%$ tested workforce vs $69 \%$ total workforce). Race/ethnicity differed between the study participants, $\mathrm{H}+\mathrm{H}$ staff completing serology testing, and the total $\mathrm{H}+\mathrm{H}$ workforce, with study participants over-representing white employees and under-representing black employees (online supplemental efigure 1A-C).

Of the 727 participants included in the study, 658 $(91 \%)$ participants completed an antibody test directly at an $\mathrm{H}+\mathrm{H}$ site, and $69(9.5 \%)$ participants reported antibody tests from an outside location. Overall, 214 (29\%) participants tested positive or reported testing positive for the presence of SARS-CoV-2 antibodies. Comparatively, of the $19107 \mathrm{H}+\mathrm{H}$ staff who completed serology testing between 30 April and 30 June, 4610 (24\%) tested positive for the presence of SARS-CoV-2 antibodies. Online supplemental efigure A-C shows seropositivity of survey participants and $19107 \mathrm{H}+\mathrm{H}$ staff by sex, race/ethnicity and age.

When comparing respondents by SARS-CoV-2 antibody status (SARS-CoV-2 IgG positive or negative), both groups were majority female (378 IgG- $(75 \%)$ vs $164 \mathrm{IgG}+(78 \%))$ (table 1 ), similar in age (mean (SD), $45^{12}$ years IgG- vs $46^{11}$ years $\mathrm{IgG}+$ ) (online supplemental etable 1 ), and majority born in the USA (301 IgG- (61\%) vs $121 \mathrm{IgG}+(58 \%))$. Compared with those who were negative for SARS-CoV-2 antibodies, a larger percentage of respondents positive for SARS-CoV-2 antibodies were black (54 IgG+ $(25 \%)$ vs $74 \mathrm{IgG}-(15 \%)$ ) (table 2), had someone in their household with COVID-19 symptoms (104 IgG+ (49\%) vs 106 IgG- $(21 \%))$, and had a COVID-19 confirmed case in their household (52 IgG+ $(25 \%)$ vs $24 \mathrm{IgG}-(5 \%))$. A lower percentage of respondents positive for SARS-CoV-2 antibodies had a clinical occupation (122 IgG+ $(64 \%)$ vs 369 IgG- $(78 \%))$, worked on a COVID-19 patient floor (58 IgG+ $(27 \%)$ vs $185 \mathrm{IgG}-(36 \%))$, in the intensive care unit (ICU) (43 IgG+ (20\%) vs 143 IgG- $(28 \%))$ or had 
Table 1 Demographics, case exposure and occupational setting by SARS-CoV-2 antibody status

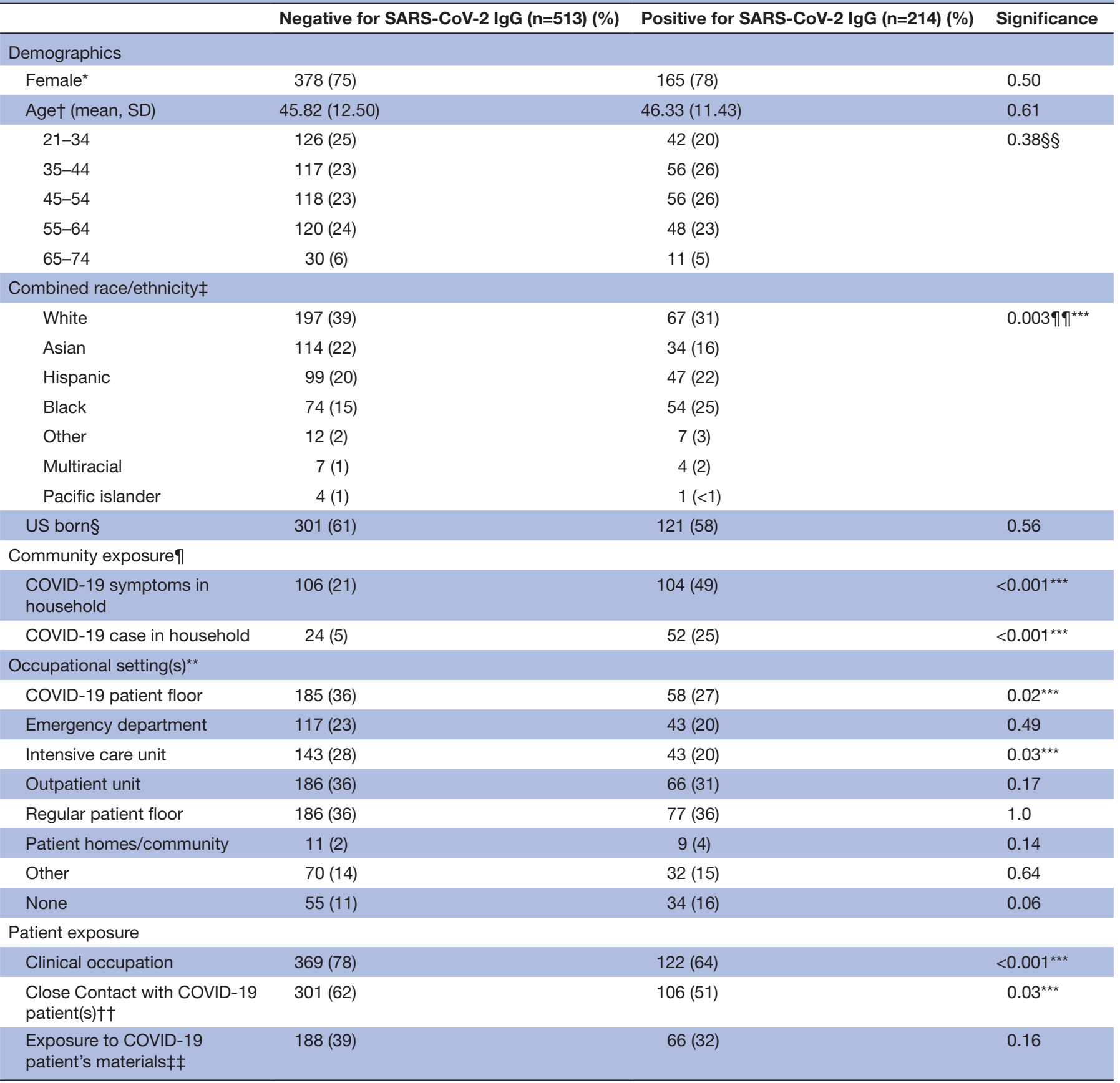

${ }^{*}$ Twelve participants were missing a response for sex in the survey resulting in a denominator of 715 participants.

tThree participants were missing a response for age in the survey resulting in a denominator of 724 participants.

$\ddagger$ Six participants were missing a response for race in the survey resulting in a denominator of 721 participants.

§Twenty-two participants were missing a response for country of origin in the survey resulting in a denominator of 705 participants.

१Ten participants were missing a response for community exposure in the survey resulting in a denominator of 717 participants.

** Participants were asked to select all settings in which they interact with patients. Two participants did not complete this question resulting in a denominator of 725 . Selecting 'None' indicates that while the participant worked for the health system, they were not working in a patient-facing setting during the study period.

††Thirty-one participants were missing a response for whether they had known close contact with a SARS-CoV-2 patient resulting in a denominator of 696 participants. This question included yes, no and unknown as response types.

抽irty-four participants were missing a response for whether they had known exposure to a SARS-CoV-2 patient's materials resulting in a denominator of 693 participants.

$\S \S$ The Fisher's exact test for age collapsed ages 55-64 and 64-74 into one category for ages 55 and over.

ๆๆThe Fisher's exact test for race collapsed multiracial and pacific islander into the other category.

***Indicates significance at level of $p<0.05$. 
Table 2 Exploratory post hoc analyses of combined race/ethnicity by SARS-CoV-2 antibody status

\begin{tabular}{llll}
\hline Combined race/ethnicity & $\begin{array}{l}\text { Negative for SARS-CoV-2 IgG } \\
(\mathbf{n = 5 1 3 )}(\%)\end{array}$ & $\begin{array}{l}\text { Positive for SARS-CoV-2 IgG } \\
(\mathbf{n = 2 1 4 )}(\%)\end{array}$ & Significance \\
\hline \\
White & $197(39)$ & $67(31)$ & Ref \\
Asian & $114(22)$ & $34(16)$ & 0.63 \\
Hispanic & $99(20)$ & $47(22)$ & 0.17 \\
Black & $74(15)$ & $54(25)$ & $0.001^{*}$ \\
Other & $23(4)$ & $12(6)$ & 0.31 \\
\hline
\end{tabular}

*Indicates significance at level of $p<0.05$.

close contact with a patient with COVID-19 (106 IgG+ $(51 \%))$ vs $301 \mathrm{IgG}-(62 \%))$.

Seven hundred and twenty respondents provided selfreported staff occupation. Self-reported occupations included 209 (28\%) doctors, nurse practitioners (NPs) or physician assistants (PAs), 154 (21\%) registered nurses (RNs), $87(12 \%)$ administrators, $15(2.1 \%)$ care coordinators, $15(2.1 \%)$ pharmacy staff, $12(1.7 \%)$ radiology staff and $8(1.1 \%)$ in food services. Crude seropositivity rates for doctors, NPs and PAs (34 IgG+) was 16\%, compared with $34 \%$ for RNs (52 IgG+), 39\% for administrators (34 IgG+) and $62 \%$ for food services ( $5 \mathrm{IgG}+$ ) (figure 1 ).

The majority of respondents reported 'always' or 'most of the time' wearing a medical or surgical mask when indicated (681 respondents (98\%)) (figure 2), with 627 (92.6\%) reporting that they were available 'always' or 'most of the time' (figure 3). A total of 430 respondents reported 'always' or 'most of the time' (72\%) wearing a respiratory mask (eg, N95), with 169 (28\%) 'never', 'rarely' or 'occasionally' wearing the respiratory mask when indicated. The availability of the respiratory masks varied, with $180(30 \%)$ saying it was 'always' available, and 235 (39\%) reporting them available 'most of the time'. The crude seropositivity rates among the 169 that 'never', 'rarely' or 'occasionally' wore respiratory masks was $36.7 \%$, compared with $26.3 \%$ for the 430 that wore a respiratory mask 'always' or 'most of the time'. For all other PPE, the majority of respondents 'always' wore PPE when indicated, except for impermeable gowns, coverall/ body suits, shoe covers and high efficiency particulate air (HEPA) filters on endotracheal tube for intubated patients. Availability of PPE varied by PPE type, with most being available 'always' or 'most of the time,' except coverall/body suits.

The distribution of seropositive study participants by borough of primary residence in NYC was highest in Queens

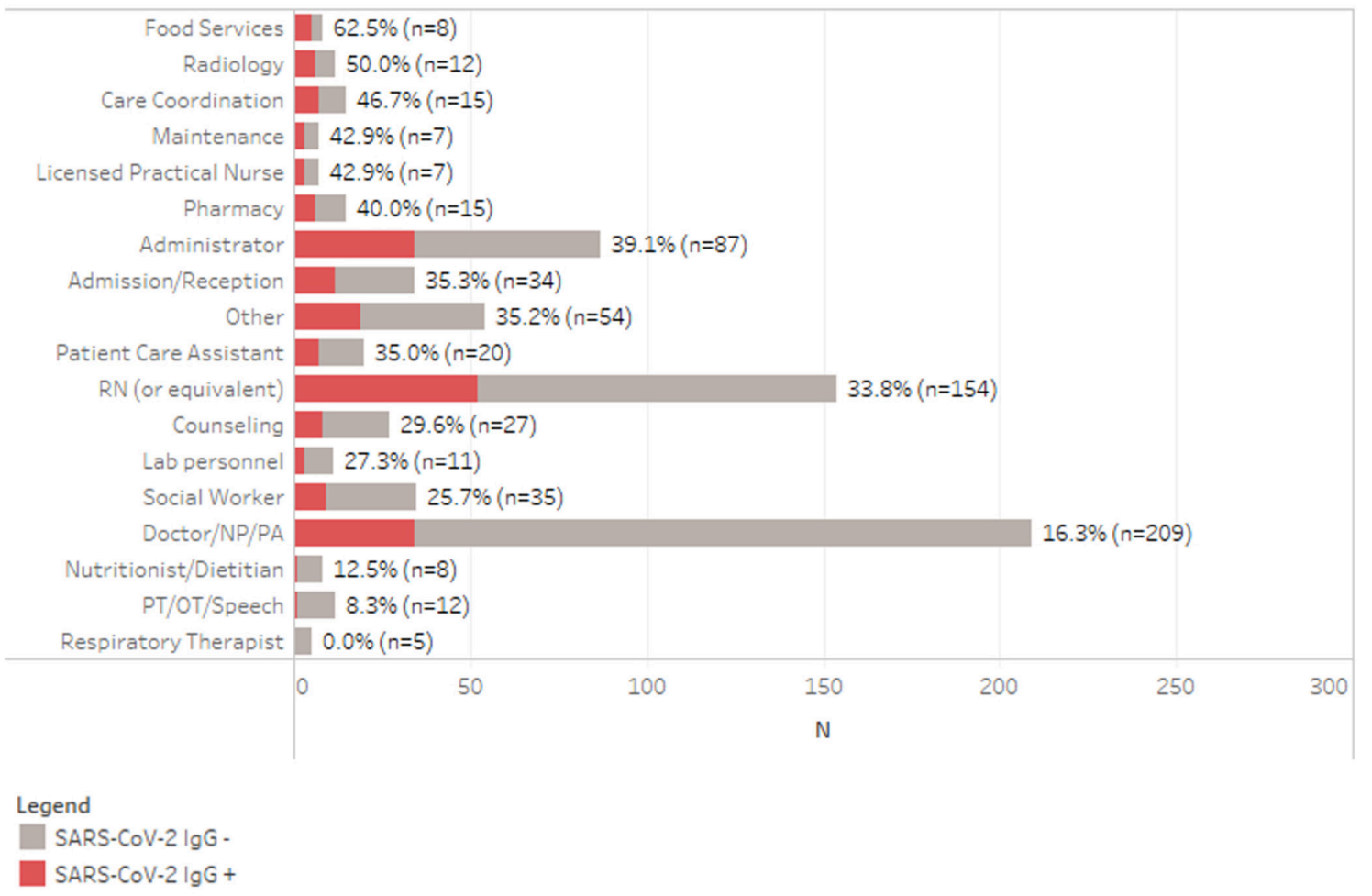

Figure 1 Per cent seropositive for SARS-CoV-2-IgG by reported staff occupation ( $n=720)$. NP, nurse practitioner; PA, physician assistant; RN, registered nurse; PT, physical therapy; OT, occupational therapy. 
Do you use this PPE when indicated?

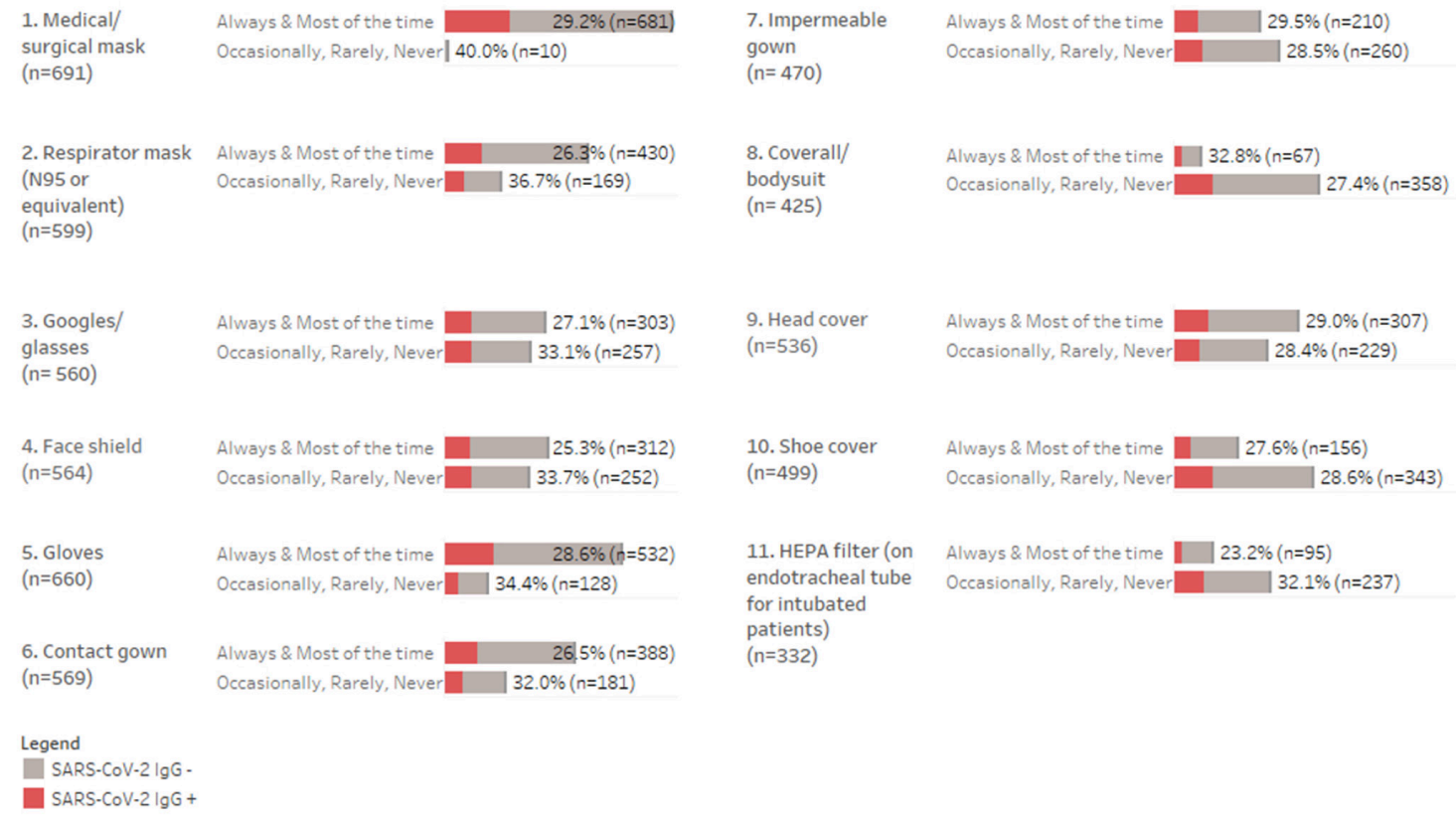

Excludes patients who noted PPE type was Not Applicable or missing.

Figure 2 Per cent seropositivity for SARS-CoV-2-IgG by how frequently PPE was used when indicated. PPE, personal protective equipment.

(34\%), followed by Brooklyn (25\%) and the Bronx (20\%). The distribution of cumulative cases reported in NYC from 29 February 2020 to 30 June 2020 was also highest in Queens $(30 \%)$, followed by Brooklyn (28\%) and the Bronx (22\%) (online supplemental etable 2).

\section{DISCUSSION}

In this system-wide survey of employees during the first wave of the COVID-19 epidemic in NYC, 29\% of study participants tested positive for SARS-CoV-2 antibodies. This was similar to

Is this PPE available in sufficient quantity in the health care facility?

\begin{tabular}{|c|c|c|c|c|}
\hline $\begin{array}{l}\text { 1. Medical/ } \\
\text { surgical mask } \\
(n=677)\end{array}$ & $\begin{array}{l}\text { Always \& Most of the time } \\
\text { Occasionally, Rarely, Never } 7.4 \%(n=50)\end{array}$ & $\begin{array}{l}\text { 7. Impermeable } \\
\text { gown } \\
(n=437)\end{array}$ & $\begin{array}{l}\text { Always \& Most of the time } \\
\text { Occasionally, Rarely, Never }\end{array}$ & $\begin{array}{l}65.7 \%(n=287) \\
34.3 \%(n=150)\end{array}$ \\
\hline $\begin{array}{l}\text { 2. Respirator mask } \\
\text { (N95 or } \\
\text { equivalent) } \\
(n=602)\end{array}$ & \begin{tabular}{l|l} 
Always \& Most of the time & $68.9 \%(n=415)$ \\
Occasionally, Rarely, Never & $31.1 \%(n=187)$
\end{tabular} & $\begin{array}{l}8 . \text { Coverall/ } \\
\text { bodysuit } \\
(n=380)\end{array}$ & $\begin{array}{l}\text { Always \& Most of the time } \\
\text { Occasionally, Rarely, Never }\end{array}$ & $\begin{array}{l}35.0 \%(n=133) \\
65.0 \%(n=247)\end{array}$ \\
\hline $\begin{array}{l}\text { 3. Googles/ } \\
\text { glasses } \\
(n=527)\end{array}$ & $\begin{array}{l}\text { Always \& Most of the time } \quad 58.8 \%(n=310) \\
\text { Occasionally, Rarely, Never }\end{array}$ & $\begin{array}{l}\text { 9. Head cover } \\
(n=501)\end{array}$ & $\begin{array}{l}\text { Always \& Most of the time } \\
\text { Occasionally, Rarely, Never }\end{array}$ & 33.3\%(n=167) \\
\hline $\begin{array}{l}\text { 5. Gloves } \\
(n=636)\end{array}$ & $\begin{array}{l}\text { Always \& Most of the time } \quad 95.0 \%(n=604) \\
\text { Occasionally, Rarely, Never } 5.0 \%(n=32)\end{array}$ & $\begin{array}{l}\text { 11. HEPA filter } \\
\text { (on endotracheal } \\
\text { tube for } \\
\text { intubated } \\
\text { patients) } \\
(n=279)\end{array}$ & $\begin{array}{l}\text { Always \& Most of the time } \\
\text { Occasionally, Rarely, Never }\end{array}$ & $\begin{array}{l}57.7 \%(n=161) \\
42.3 \%(n=118)\end{array}$ \\
\hline $\begin{array}{l}\text { 6. Contact gown } \\
(n=557)\end{array}$ & \begin{tabular}{l|r|} 
Always \& Most of the time & $84.9 \%(n=473)$ \\
Occasionally, Rarely, Never & $15.1 \%(n=84)$
\end{tabular} & & & \\
\hline
\end{tabular}

Excludes patients who noted PPE type was Not Applicable or missing.

Figure 3 PPE availability. PPE, personal protective equipment. 
the overall seropositivity of employees tested at $\mathrm{NYCH}+\mathrm{H}$, and among the highest rates of employee seropositivity reported by health systems in the NYC area during this period. ${ }^{16}{ }^{17}$ One possible difference to explain these higher rates of seropositivity at $\mathrm{NYC} \mathrm{H}+\mathrm{H}$ compared with rates reported from health systems in Manhattan and Long Island could be the higher rates of community exposure at the time in Queens, Brooklyn and the Bronx, where the majority of $\mathrm{NYC} \mathrm{H}+\mathrm{H}$ employees live. Study participant seropositivity was highest for employees living in Queens, Brooklyn and the Bronx. And while citywide seroprevalence data by borough was not yet available for direct comparison during this time period, the cumulative distribution of cases in NYC at the time closely followed this trend. Employee seropositivity may have been largely driven by community spread at this phase of the pandemic in NYC, as seropositivity was most strongly associated with household COVID-19 contact. However, a lack of temporal data limits whether this might be interpreted as community transmission to HCW or HCW transmitting to household members.

Doctor/NP/PA were the most frequently reported occupations among respondents, and they were among the lowest in seropositivity. This may reflect differing patient exposures or differing use of PPE, as RNs (or equivalent) were the second largest occupational group surveyed and had over double the seropositivity rate. A higher seropositivity for RNs was also noted in a larger study of 10662 hospital staff in the UK, where seroprevalence was highest among nurses and healthcare assistants. ${ }^{12}$ Similarly, in a systematic review of COVID-19 infection in HCW up through July of 2020, the largest occupational group of HCW testing positive for COVID-19 were nurses. ${ }^{7}$ As the duties of RNs involve frequent contact with patients for tasks such as medication administration and other beside functions, these results suggest occupational exposure is a significant risk factor for infection. However, seropositivity rates were also higher among other large occupational groups surveyed such as administrator and admission/ reception, which is notable as these are patient-facing but typically non-clinical occupation groups. In fact, working on a COVID-19 patient floor or ICU was associated with testing negative for SARS-CoV-2 antibodies, as was close contact with COVID-19 patients and being employed in a clinical occupation. These results potentially indicate greater adherence to PPE, greater availability of PPE or clearer recommendations on PPE usage for providers working in those settings, which offset patient exposure.

In examining employee use of PPE, consistent use of certain equipment was associated with lower reported rates of seropositivity. Most notably, employees who reported using an N95 mask 'always' or 'most of the time' when indicated had a seropositivity rate of $26.3 \%$, while employees using an N95 'occasionally', 'rarely' or 'never' had seropositivity rates of $36.7 \%$. Although a majority $(58 \%)$ of respondents reported always wearing an N95 when indicated, only 30\% reported an N95 was always available in sufficient quantity in the healthcare facility. This not only reflects the low supply of PPE in NYC at the height of the first wave of the pandemic, but also the shortfall in PPE that HCW needed to bridge by stretching available supplies. Practices to stretch PPE supply reported elsewhere in the literature included extended use, reuse, or decontamination procedures, and may account for this disparity in responses. ${ }^{18} 19$

Black race was strongly associated with employee seropositivity, echoing broader racial inequities seen in the community during the COVID-19 pandemic. ${ }^{20}{ }^{21}$ In the USA, among 100570 cases of SARS-CoV-2 and 641 deaths among HCW up to 16 July 2020 that were reported to the Centers for Disease Control and Prevention, Asian and black HCW were over-represented among fatal cases, and healthcare support workers, nurses and administrative staff were the most common occupational types among those infected. ${ }^{22}$ In a prospective cohort study using selfreported data through a smartphone application among almost 100000 HCW in the UK and USA, Asian, black and other minority ethnic HCW were at higher risk of SARS-CoV-2 infection and more likely to report having inadequate access to PPE compared with white $\mathrm{HCW}^{23}$ These results underscore the connection between HCW and their communities with regard to these systemic inequities, and highlight the continued need to urgently address these disparities for the well-being of patients as well as the workers caring for those patients.

There were several limitations to our study. First, convenience sampling was used to enrol study participants, with less than $10 \%$ of employees with antibody testing results having completed our survey. And while we did not record reasons for non-response to the survey, we recruited study participants from a voluntary universal screening programme, and there was a potential for selection bias in terms of occupation type and level of exposure. There may have also been socioeconomic factors related to participation that could be strongly tied to demographic and occupational characteristics. We attempted to partially mitigate these limitations by comparing aggregate participant demographics with all employees undergoing antibody testing, as well as with overall NYC $\mathrm{H}+\mathrm{H}$ employee demographics. This revealed under-representation of certain groups in the study participants, most notably black employees. Given that black employees were also found to be more likely to have SARS-CoV-2 antibodies, this may have skewed overall positivity rates and excluded differing exposure factors. We were also unable to determine when employees with positive SARS-CoV-2 antibodies were infected. And with continuously changing guidelines around PPE during the initial surge, it is difficult to link exposure and infection with evolving PPE practices. Furthermore, employees who were sick or were suspected to have SARS-CoV-2 may have been less likely to get serological testing, and participants who previously tested PCR positive for SARS-CoV-2 may or may not have chosen serological testing. Our study did not account for HCW who were currently hospitalised or had died. These factors may have further biased our sample and underestimated the burden of SARS-CoV-2 infection in this population. Sixty-nine respondents self-reported outside 
serology results, and while they made up a small proportion of total respondents, we were unable to adjust results of non-standardised serology testing due to limitations of study design, Finally, this was a descriptive analysis and it is possible that some findings may be due uncontrolled confounding factors.

Strengths of this study included the sampling of a wide range of employees and healthcare occupations, including both clinical and non-clinical occupations. This study also used a detailed survey that allowed for the stratification of SARS-CoV-2 serostatus by a wide range of demographic, occupational and exposure factors. Finally, this study included detailed questions on PPE use and availability, allowing for further description of serostatus by self-reported PPE use and availability during the first wave of the COVID-19 pandemic.

\section{CONCLUSION}

Employees at a large, public hospital system reported a seropositivity rate of $29 \%$ during the first wave of the COVID-19 epidemic in NYC. This was among the highest employee seropositivity rates reported in NYC, and the risk of exposure varied significantly by employee demographics, occupation and work location. Results underscore the need to address exposure risks for HCW across occupational settings, including appropriate PPE, as well as address broader inequities at the community level where HCW live.

\section{Author affiliations}

${ }^{1}$ Department of Medicine, NYU Grossman School of Medicine, New York, New York, USA

${ }^{2}$ Office of Ambulatory Care and Population Health, NYC Health + Hospitals, New York, New York, USA

${ }^{3}$ Department of Medicine, NYC Health + Hospitals/Woodhull, Brooklyn, New York, USA

${ }^{4}$ Department of Global Health and Health System Design, Icahn School of Medicine at Mount Sinai, New York, New York, USA

${ }^{5}$ Department of Surgery, Memorial Sloan Kettering Cancer Center, New York, New York, USA

${ }^{6}$ Arnhold Institute for Global Health, Icahn School of Medicine at Mount Sinai, New York, New York, USA

${ }^{7}$ Center for Global Health, Indiana University, Bloomington, Indiana, USA

${ }^{8}$ Department of Medicine, NYC Health + Hospitals/Jacobi, New York, New York, USA

${ }^{9}$ Department of Global Health, NYC Health + Hospitals/Elmhurst, Elmhurst, New

York, USA

${ }^{10}$ Department of Medicine, Icahn School of Medicine at Mount Sinai, New York, New

York, USA

${ }^{11}$ Department of Medicine, NYC Health + Hospitals/Queens, Jamaica, New York, USA

${ }^{12}$ Department of Pediatrics, Icahn School of Medicine at Mount Sinai, New York, New York, USA

${ }^{13}$ Departments of Global Health, Internal Medicine, and Pediatrics, Icahn School of

Medicine at Mount Sinai, New York, New York, USA

${ }^{14}$ Departments of Medicine and Pediatrics, NYC Health + Hospitals/Elmhurst, Elmhurst, New York, USA

Twitter Duncan Maru @duncanmaru

Contributors All authors contributed substantially to the development of the research plan, analysis, and/or writing of this paper. NJD, JM, AR and DM conceived and designed the study. JD-G and AB led data collection. KT and CC provided statistical analysis. MS, KT, CC and AB drafted the manuscript, with support from
$A R, N C, P K, E S, P B, S P, N J D$ and DM. AB is responsible for the overall content as the guarantor.

Funding The authors have not declared a specific grant for this research from any funding agency in the public, commercial or not-for-profit sectors.

Competing interests None declared.

Patient consent for publication Not applicable.

Ethics approval This study was approved by the Institutional Review Board of the Biomedical Research Alliance of New York, BRANY IRB File \# 20-12-175-373(HHC).

Provenance and peer review Not commissioned; externally peer reviewed.

Data availability statement Data are available on reasonable request. Deidentified data are available upon reasonable request from the corresponding author. For requests, contact albryan16@gmail.com.

Supplemental material This content has been supplied by the author(s). It has not been vetted by BMJ Publishing Group Limited (BMJ) and may not have been peer-reviewed. Any opinions or recommendations discussed are solely those of the author(s) and are not endorsed by BMJ. BMJ disclaims all liability and responsibility arising from any reliance placed on the content. Where the content includes any translated material, BMJ does not warrant the accuracy and reliability of the translations (including but not limited to local regulations, clinical guidelines, terminology, drug names and drug dosages), and is not responsible for any error and/or omissions arising from translation and adaptation or otherwise.

Open access This is an open access article distributed in accordance with the Creative Commons Attribution Non Commercial (CC BY-NC 4.0) license, which permits others to distribute, remix, adapt, build upon this work non-commercially, and license their derivative works on different terms, provided the original work is properly cited, appropriate credit is given, any changes made indicated, and the use is non-commercial. See: http://creativecommons.org/licenses/by-nc/4.0/.

ORCID iDs

Alexander Bryan http://orcid.org/0000-0002-4497-5438

Duncan Maru http://orcid.org/0000-0002-5923-6910

\section{REFERENCES}

1 Dong E, Du H, Gardner L. An interactive web-based dashboard to track COVID-19 in real time. Lancet Infect Dis 2020;20:533-4.

2 Suwantarat N, Apisarnthanarak A. Risks to healthcare workers with emerging diseases: lessons from MERS-CoV, Ebola, SARS, and avian flu. Curr Opin Infect Dis 2015;28:349-61.

3 The Lancet. COVID-19: protecting health-care workers. Lancet 2020;395:922.

4 World Health Organization. Protocol for assessment of potential risk factors for 2019-novel coronavirus (2019-nCoV) infection among health care workers in a health care setting. Geneva, 2020.

5 Wang D, Hu B, Hu C, et al. Clinical characteristics of 138 hospitalized patients with 2019 novel coronavirus-infected pneumonia in Wuhan, China. JAMA 2020;323:1061.

6 Bandyopadhyay S, Baticulon RE, Kadhum M, et al. Infection and mortality of healthcare workers worldwide from COVID-19: a systematic review. BMJ Glob Health 2020;5:e003097.

7 Gómez-Ochoa SA, Franco OH, Rojas LZ, et al. COVID-19 in healthcare workers: a living systematic review and meta-analysis of prevalence, risk factors, clinical characteristics, and outcomes. Am J Epidemiol 2021:190:161-75.

8 Sommerstein R, Fux CA, Vuichard-Gysin D, et al. Risk of SARSCoV-2 transmission by aerosols, the rational use of masks, and protection of healthcare workers from COVID-19. Antimicrob Resist Infect Control 2020;9:100.

9 Heinzerling A, Stuckey MJ, Scheuer T, et al. Transmission of COVID-19 to health care personnel during exposures to a hospitalized patient - Solano County, California, February 2020. MMWR Morb Mortal Wkly Rep 2020;69:472-6.

10 Reusken CB, Buiting A, Bleeker-Rovers C, et al. Rapid assessment of regional SARS-CoV-2 community transmission through a convenience sample of healthcare workers, the Netherlands, March 2020. Euro Surveill 2020;25.

11 Shah ASV, Wood R, Gribben C, et al. Risk of hospital admission with coronavirus disease 2019 in healthcare workers and their households: nationwide linkage cohort study. BMJ 2020;371:m3582.

12 Martin CA, Patel P, Goss C, et al. Demographic and occupational determinants of anti-SARS-CoV-2 IgG seropositivity in hospital staff. 
J Public Health 2020. doi:10.1093/pubmed/fdaa199. [Epub ahead of print: 16 Nov 2020].

13 Baker MG, Peckham TK, Seixas NS. Estimating the burden of United States workers exposed to infection or disease: a key factor in containing risk of COVID-19 infection. PLoS One 2020;15:e0232452.

14 US Food and Drug Administration. Policy for diagnostic tests for coronavirus disease-2019 during the public health emergency, 2020.

15 U.S. Food and Drug Administration. Policy for coronavirus disease-2019 tests during the public health emergency (revised), 2020.

16 Moscola J, Sembajwe G, Jarrett M, et al. Prevalence of SARS-CoV-2 antibodies in health care personnel in the New York City area. JAMA 2020;324:893-5.

17 Mansour M, Leven E, Muellers K, et al. Prevalence of SARS-CoV-2 antibodies among healthcare workers at a tertiary academic hospital in New York City. J Gen Intern Med 2020;35:2485-6.

18 Schwartz A, Stiegel M, Greeson N, et al. Decontamination and reuse of $\mathrm{N} 95$ respirators with hydrogen peroxide vapor to address worldwide personal protective equipment shortages during the SARS-CoV-2 (COVID-19) pandemic. Applied Biosafety 2020;25:67-70.

19 Livingston E, Desai A, Berkwits M. Sourcing personal protective equipment during the COVID-19 pandemic. JAMA 2020;323:1912-4.

20 Egede LE, Walker RJ. Structural racism, social risk factors, and Covid-19 - a dangerous convergence for black Americans. N Engl J Med Overseas Ed 2020;383:e77.

21 Lopez L, Hart LH, Katz MH. Racial and ethnic health disparities related to COVID-19. JAMA 2021;325:719.

22 Hughes MM, Groenewold MR, Lessem SE, et al. Update: characteristics of health care personnel with COVID-19 - United States, February 12-July 16, 2020. MMWR Morb Mortal Wkly Rep 2020;69:1364-8.

23 Nguyen LH, Drew DA, Graham MS, et al. Risk of COVID-19 among front-line health-care workers and the general community: a prospective cohort study. Lancet Public Health 2020;5:e475-83. 